\title{
Death Following Recreational Use of Designer Drug "Bath Salts" Containing 3,4-Methylenedioxypyrovalerone (MDPV)
}

\author{
Brittany L. Murray • Christine M. Murphy • \\ Michael C. Beuhler
}

Published online: 20 January 2012

(C) American College of Medical Toxicology 2012

\begin{abstract}
Introduction 3,4-Methylenedioxypyrovalerone (MDPV) is a designer stimulant drug that has gained popularity in the USA. Although adverse effects of MDPV have been described, to our knowledge, this is the first reported death.

Case Report We report the case of a 40-year-old male who injected and snorted "bath salts" containing MDPV and subsequently became agitated, aggressive, and experienced a cardiac arrest. He was resuscitated after his initial arrest; however, he developed hyperthermia, rhabdomyolysis, coagulopathy, acidosis, anoxic brain injury, and subsequently died.

Discussion This is the first case in the medical literature to report death due to isolated confirmed MDPV intoxication. The manner of death is also consistent with excited delirium syndrome.
\end{abstract}

Keywords 3,4-Methylenedioxypyrovalerone - Bath salts . Excited delirium syndrome $\cdot \mathrm{MDPV} \cdot$ Designer drug

\section{Introduction}

Psychoactive products containing $\beta$-keto phenylalkylamines such as 3,4-methylenedioxypyrovalerone (MDPV), mephedrone, and methylone have entered the recreational

B. L. Murray · C. M. Murphy $(\bowtie)$

Department of Emergency Medicine, Carolinas Medical Center, PO Box 32861, MEB 3rd Floor,

Charlotte, NC 28232, USA

e-mail: christine.murphy66@gmail.com

M. C. Beuhler

Carolinas Poison Center,

Charlotte, NC, USA drug market over the past several years. These products are often labeled as "bath salts" or "plant food," with the disclaimer that they are "not for human consumption" to skirt state and federal laws. Although states are creating new laws to address these derivatives, an increasing number of exposures have been reported to United States (US) poison centers since late 2010 [1].

MDPV is a ring-substituted analogue of pyrovalerone (Fig. 1). Pyrovalerone is a stimulant and a schedule V controlled substance that was first synthesized in 1964. The synthesis of MDPV was first reported in 1969 [2, 3]. The chemical structure of MDPV is similar to methcathinone ("Mcat") and to hallucinogenic substances like 3,4-methylenedioxymethamphetamine (MDMA or "Ectasy"), but it is best characterized as a $\beta$-keto phenylalkylamine (Fig. 1). Over the past decade, MDPV has gained popularity as a designer drug, or "legal high," across Europe. The first designer drug containing MDPV was identified in Germany in 2007 [4]. In Japan, MDPV was retrospectively identified in designer drugs confiscated in the year 2006 [5]. The recreational use of MDPV in the USA has become more prevalent since late 2010 and it is now illegal in many states [1]. We report the first case of isolated recreational use of MDPV resulting in excited delirium syndrome and ultimately death, with confirmatory toxicological analyses.

\section{Case Report}

A 40-year-old man with a history of bipolar disorder snorted and injected an unknown amount of "bath salts." Family and friends reported he had recently switched from abusing cocaine to using "bath salts" products. Shortly after his consumption of this product, he became aggressive, uncontrollable, delusional, removed all of his clothing, and 
Fig. 1 Chemical structures of 3,4-methylenedioxypyrovalerone (MDPV); cathinone; 3,4-methylenedioxymethamphetamine (MDMA); and pyrovalerone<smiles>CCCC(C(=O)c1ccc(C)cc1)N1CCCC1</smiles>

ran outside. Police were called and while being taken into custody, the patient displayed aggression, considerable strength, and violent behavior. An electronic control device was discharged three times in an effort to overpower him and protect others on scene. During ambulance transport, he remained aggressive and delusional and was physically restrained. He was noted to have slightly labored breathing and was placed on a non-rebreather mask (NRB) with $100 \%$ oxygen. He was yelling incomprehensibly and was noted to have dilated pupils. Initial vital signs in the prehospital setting were as follows: heart rate 164 beats per minute (bpm); blood pressure $131 / 72 \mathrm{mmHg}$; respiratory rate 24 breaths/min, and oxygen saturation of $100 \%$ on NRB. Prehospital electrocardiogram (EKG) initially demonstrated sinus tachycardia with widened QTc interval and peaked T waves (Fig. 2a). Repeat EKG 10 min later depicted normal sinus rhythm with persistent peaked $\mathrm{T}$ waves (Fig. 2b). Sedation was attempted unsuccessfully with $2 \mathrm{mg}$ of intramuscular lorazepam.

Upon arrival in the hospital, he continued with very aggressive behavior and incomprehensible screaming. A review of the patient's electronic medical records revealed previous routine medications of quetiapine, methadone, temazepam, and 10/650 mg hydrocodone/acetaminophen. As the patient was never conversant, compliance was not established. Vital signs at the time of his arrival, $15 \mathrm{~min}$ after reported EMS vital signs, were as follows: oral temperature 98. $0^{\circ} \mathrm{F}$; blood pressure 100/64 $\mathrm{mmHg}$; heart rate $91 \mathrm{bpm}$; respiratory rate 12 breaths/min; and oxygen saturation of $100 \%$ on NRB. While being transitioned from the Emergency Medical Services stretcher to a hospital bed, and without further intervention, he became very quiet and withdrawn.

Fig. 2 a Initial prehospital EKG. b Repeat prehospital EKG performed 10 min later demonstrating normal sinus rhythm with rate of $85 \mathrm{bpm}, \mathrm{PR}$ interval $110 \mathrm{msec}$, QRS interval $116 \mathrm{msec}$, QTc interval $414 \mathrm{msec}$, and peaked $\mathrm{T}$ waves

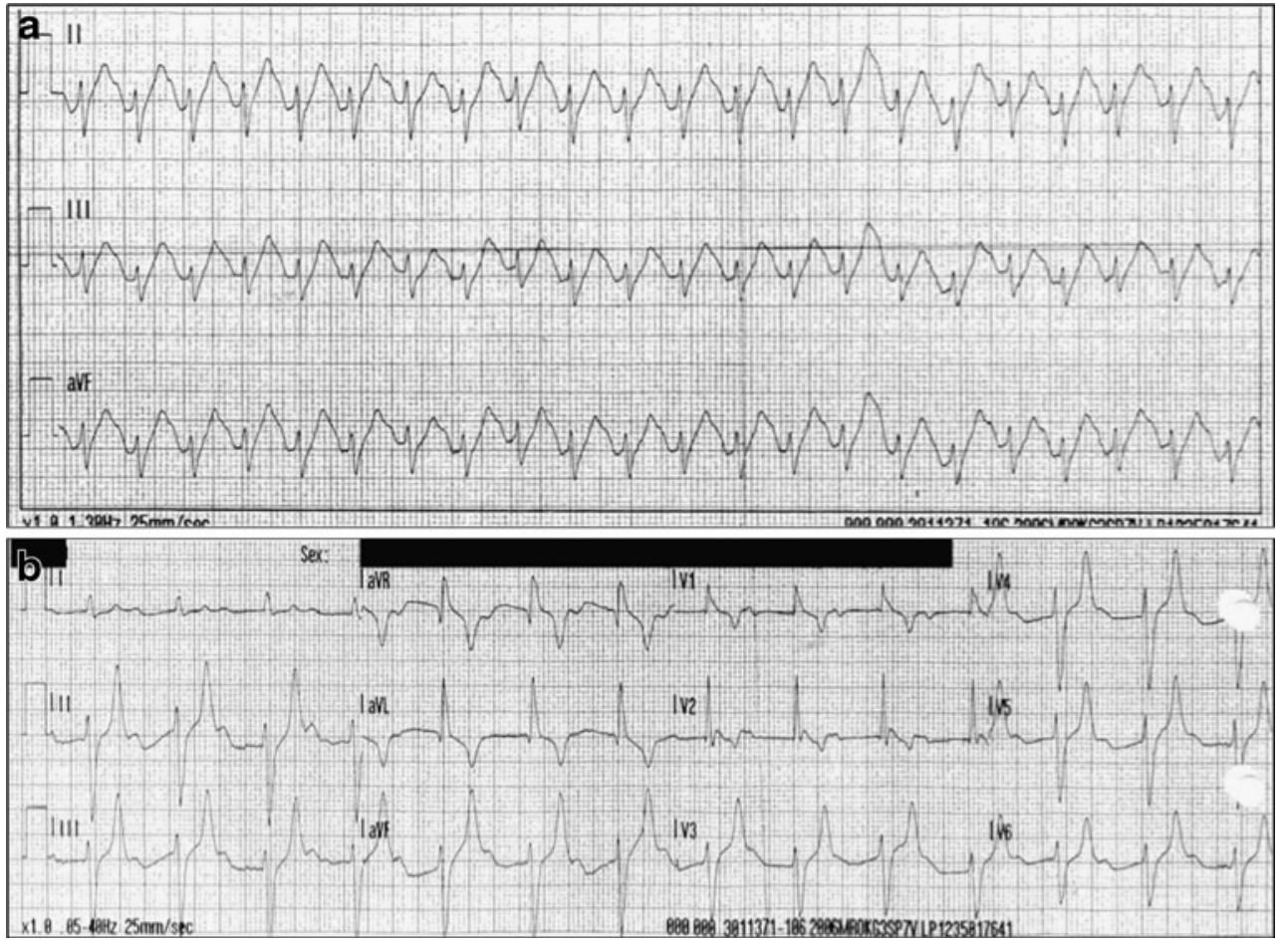


Within 5 minutes of his arrival to the hospital he developed bradycardia and subsequent cardiac arrest with pulseless electrical activity (PEA). Standard advanced cardiac life support measures were initiated including cardiopulmonary resuscitation and administration of two doses each of $1 \mathrm{mg}$ intravenous (IV) epinephrine and $1 \mathrm{mg}$ IV atropine, as well as $100 \mathrm{mg}$ of IV lidocaine. He was intubated and given both $2 \mathrm{mg}$ IV naloxone and $0.5 \mathrm{mg}$ IV flumazenil without effect. Return of spontaneous circulation was achieved after $30 \mathrm{~min}$ of resuscitation. Dopamine and phenylephrine infusions were initiated at rates of $5 \mathrm{mcg} / \mathrm{kg} / \mathrm{h}$ and $10 \mathrm{mcg} / \mathrm{h}$, respectively, due to persistent hypotension despite a bolus infusion of 2 liters $0.9 \%$ normal saline (NS).

Immediately after resuscitation, a rectal temperature was $105.4^{\circ} \mathrm{F}$, for which he was given $1,300 \mathrm{mg}$ of acetaminophen per rectum. Other vital signs included a blood pressure of 70/32 mmHg; heart rate of $91 \mathrm{bpm}$; and oxygen saturation of $100 \%$ while mechanically ventilated. Physical examination revealed a Glascow coma scale score of 3 and pupils dilated at $6 \mathrm{~mm}$ with minimal reaction to light. Skin examination revealed dry skin with multiple needle marks in both antecubital fossae. Additionally, there was no evidence of hypertonia, hyperreflexia, inducible or spontaneous clonus, or bruxism.

Pertinent initial labs in the immediate post-arrest period included: potassium of $7.4 \mathrm{mmol} / \mathrm{L}$, creatinine of $3.0 \mathrm{mg} / \mathrm{dL}$, negative serum acetaminophen and ethanol levels (limit of detection less than or equal to $5 \mathrm{mg} / \mathrm{dL}$ ), a salicylate level of $4.1 \mathrm{mg} / \mathrm{dL}$, and a urine drug screen positive for opiates but negative for cocaine, phencyclidine, amphetamine, tetrahydrocannabinol, benzodiazepines, and barbiturates. Initial international normalized ratio (INR) was 1.01; creatinine kinase $234 \mathrm{U} / \mathrm{L}$; aspartate aminotransferase (AST) $19 \mathrm{U} / \mathrm{L}$; and alanine transaminase (ALT) was $36 \mathrm{U} / \mathrm{L}$. An EKG was also obtained at this time and showed changes consistent with hyperkalemia (peaked $\mathrm{T}$ waves) with a sinus bradycardia at a rate of $56 \mathrm{bpm}$ with a markedly prolonged QRS of 240 milliseconds (msec) (Fig. 3). The patient was then transferred to a tertiary hospital with hemodialysis capabilities and a toxicology consult service.

On arrival to the tertiary care center, the patient's temperature had decreased to $100.2^{\circ} \mathrm{F}$ without further intervention. The patient's blood pressure remained low at $85 / 41 \mathrm{mmHg}$ despite elevated doses of dopamine $(20 \mathrm{mcg} / \mathrm{kg} / \mathrm{min})$, phenylephrine (180 mcg/h), and additional boluses of chilled IV NS (4 liters); therefore, norepinephrine was started $(4 \mathrm{mcg} / \mathrm{min})$. His heart rate was $115 \mathrm{bpm}$ and oxygen saturation of $100 \%$ with the patient breathing over the set ventilator rate of $20 \mathrm{breaths} / \mathrm{min}$ at $32 \mathrm{breaths} / \mathrm{min}$. His pupils remained dilated and were minimally reactive to light; however, on neurologic examination, he had a normal gag reflex, normal corneal reflexes, and he flexed in response to pain with all four extremities. Repeat EKG showed a bradycardia with a rate of $53 \mathrm{bpm}$, a right bundle branch block with hyperacute T waves, some ST depression in V2 and V3, a QRS interval of $158 \mathrm{msec}$, and QTc interval of $420 \mathrm{msec}$. A venous blood gas was performed: $\mathrm{pH} \mathrm{7.2,} \mathrm{pCO}_{2} 39 \mathrm{mmHg}$, $\mathrm{pO}_{2} 35 \mathrm{mmHg}, \mathrm{HCO}_{3} 16.2 \mathrm{mmol} / \mathrm{L}$, base excess $-11 \mathrm{mEq} / \mathrm{L}$, and serum lactate of $2.83 \mathrm{mmol} / \mathrm{L}$. Laboratory testing repeated $5 \mathrm{~h}$ after initial presentation demonstrated continued hyperkalemia $(8.0 \mathrm{mmol} / \mathrm{L})$, an elevated creatinine $(3.5 \mathrm{mg} / \mathrm{dL})$, and marked increase in AST (869 U/L), ALT (738 U/L), INR (4.2), and creatinine kinase $(14,839 \mathrm{U} / \mathrm{L})$. His hyperkalemia was treated with $1 \mathrm{~g}$ of calcium gluconate, $50 \mathrm{mEq}$ of sodium

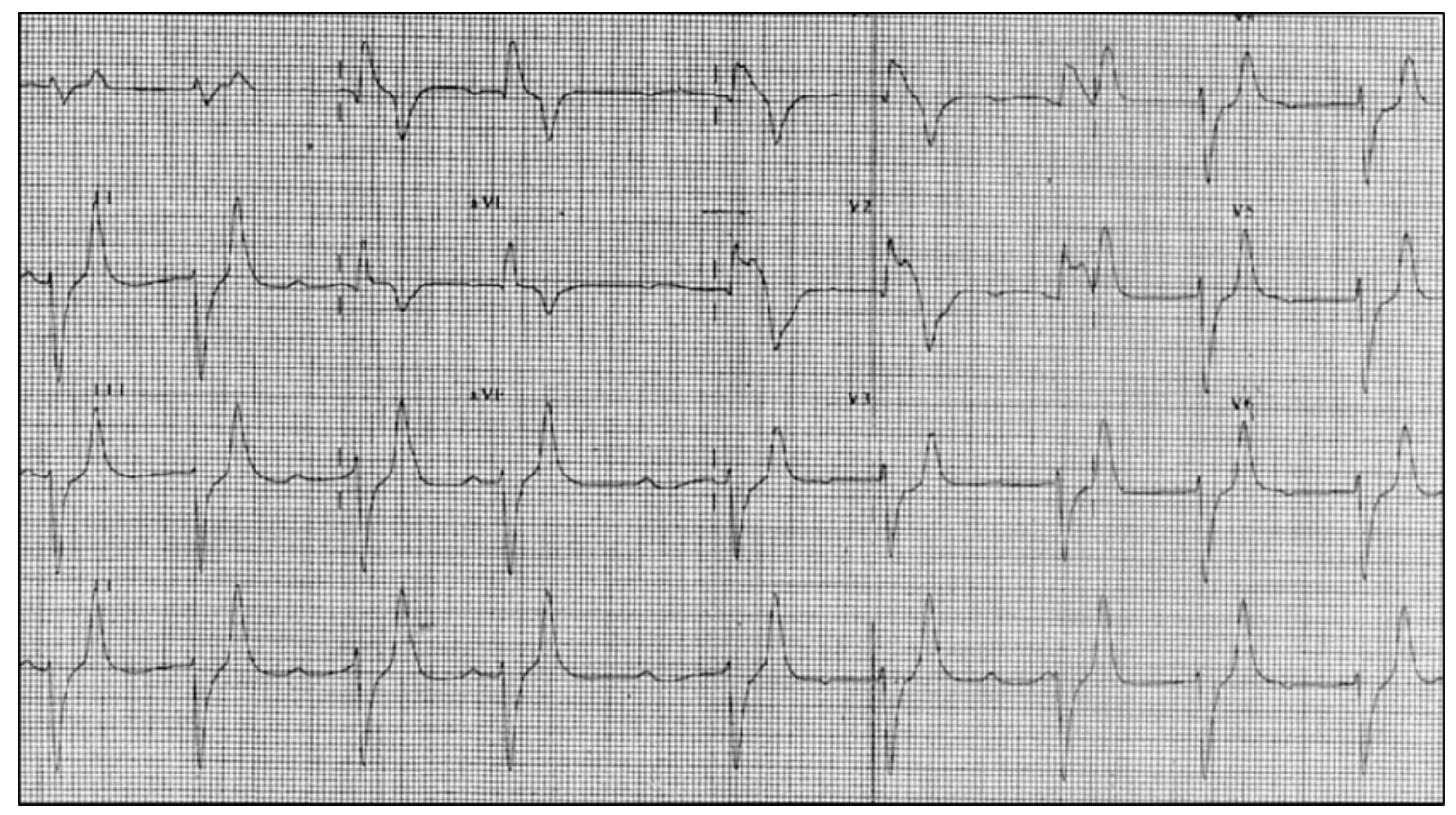

Fig. 3 Post-arrest EKG 
bicarbonate, 10 units of regular insulin, and $50 \mathrm{~g}$ of dextrose. His potassium subsequently decreased to $6.0 \mathrm{mmol} / \mathrm{L}$ and a repeat EKG demonstrated sinus tachycardia $(120 \mathrm{bpm})$ with normalization of the T waves (Fig. 4).

He was admitted to the medical intensive care unit where he was started on an infusion of $5 \%$ dextrose in water with $150 \mathrm{mEq}$ of sodium bicarbonate at a rate of $125 \mathrm{ml} / \mathrm{h}$, hydrocortisone (100 mg IV every $8 \mathrm{~h}$ ), and propofol infusion $(10 \mathrm{mcg} / \mathrm{kg} / \mathrm{h})$. Persistent hypotension was treated with an additional 2 liters of bolused NS and a vasopressin infusion was started at 0.04 units $/ \mathrm{min}$. Over the next $3 \mathrm{~h}$, he developed a worsening metabolic acidosis (arterial $\mathrm{pH}$ 7.14; $\mathrm{HCO}_{3} 10 \mathrm{mmol} / \mathrm{L}$ ) with oliguric renal failure that progressed to anuria (creatinine $4.26 \mathrm{mg} / \mathrm{dL}$ ), worsening transaminase elevation (AST 10,873 U/L; ALT 6,623 $\mathrm{U} / \mathrm{L}$ ), and rhabdomyolysis (creatinine kinase $75,952 \mathrm{U} / \mathrm{L}$ ). His coagulopathy progressed and his INR was $>9.3$ (upper limit of laboratory detection) and PT $>96 \mathrm{~s}$ (upper limit of laboratory detection). He was noted to be very pale and began having melanotic stools. He developed an anemia (hemoglobin of $6.3 \mathrm{~g} / \mathrm{dL}$ ) and thrombocytopenia with platelets of $11 \times 10^{9} / \mathrm{L}$. Hemodialysis was started $17 \mathrm{~h}$ after presentation to correct the acidosis and renal failure. Additionally, he received packed red blood cells (4 units), platelets ( 2 units), cryoprecipitate (9 units), and fresh frozen plasma (10 units). A non-contrasted brain computed tomography showed decreased gray-white matter discrimination interpreted by the radiologist as "likely edema and early anoxic injury." An EEG was performed and showed diffuse slowing consistent with coma and likely anoxic injury.

Despite improvement in his acidosis and anemia after dialysis and transfusion, his neurologic exam worsened. His pupils became fixed and dilated and he lost his gag, corneal, and vestibulo-ocular reflexes. He no longer responded to noxious stimuli. Approximately $42 \mathrm{~h}$ after his initial presentation, the patient was declared brain dead by clinical criteria and support was withdrawn.

During his evaluation, several toxicology laboratories were evaluated on blood and urine samples obtained at the time of arrival to the tertiary care center. His urine was negative for barbiturates, amphetamines, benzodiazepines, cocaine, marijuana, methadone, and opiates. Urine liquid chromatography was positive for trimethoprim. Serum ethanol levels were $11 \mathrm{mg} / \mathrm{dL}$ ( $8 \mathrm{~h}$ after presentation) and $25 \mathrm{mg} /$ $\mathrm{dL}$ (19 $\mathrm{h}$ after presentation). His qualitative urine methadone screen was negative. Serum salicylate level was $7.9 \mathrm{mg} / \mathrm{dL}$ and serum acetaminophen level was $2.9 \mathrm{mcg} / \mathrm{mL}$. A lithium level was $<0.1 \mathrm{mmol} / \mathrm{L}$. Ethanol, ethylene glycol, methanol, and isopropanol were not detected by gas chromatography 21 $\mathrm{h}$ after presentation.

Samples of the patient's urine and serum obtained at the time of his arrival to the tertiary care center were sent for further testing at NMS Labs (Forensic Science Department, Willow Grove, PA). An initial therapeutic drug screen using gas chromatography/mass spectrometry was performed and positive for acetaminophen, caffeine, cotinine, lidocaine, trimethoprim, and MDPV; quetiapine was not detected. Confirmatory testing was undertaken using high performance liquid chromatography/tandem mass spectrometry (LC/MSMS). Specimens were fortified with $d_{8}$-MDPV as an internal standard and then subjected to a single step liquid-liquid extraction using trichloroacetic acid (TCA). MDPV was quantified in his urine $(670 \mathrm{ng} / \mathrm{mL})$ and serum $(82 \mathrm{ng} / \mathrm{mL})$. Trimethoprim was also quantified using high performance thin layer chromatography $(12 \mathrm{mcg} / \mathrm{mL}$ in urine and $2.2 \mathrm{mcg} / \mathrm{mL}$ in serum).

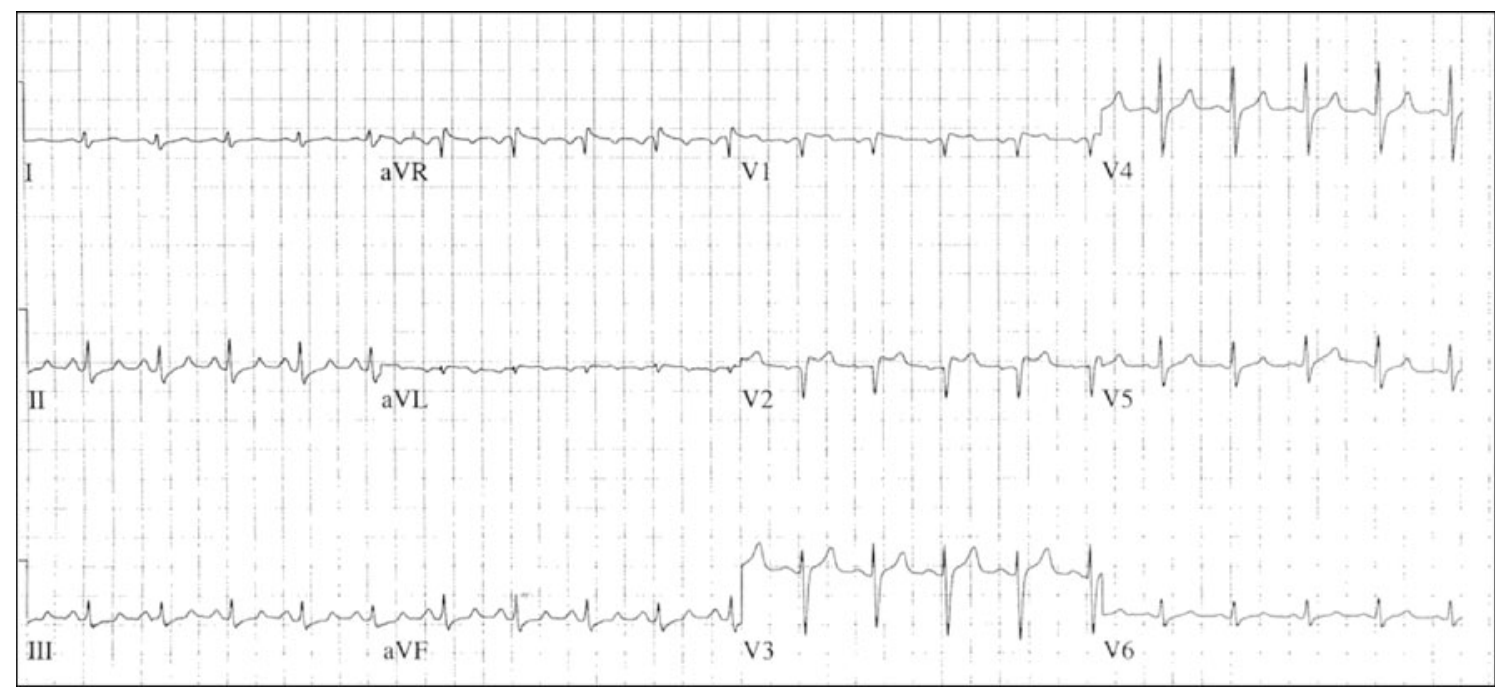

Fig. 4 EKG after treatment for hyperkalemia. PR interval of $125 \mathrm{msec}$, QRS interval of $83 \mathrm{msec}$, QTc interval of $412 \mathrm{msec}$, normalization of the T waves and ST depressions in leads II and III with mild ST elevation in leads V1 and V2 


\section{Discussion}

MDPV is a white or tan powder that is insufflated, injected intravenously, or ingested. Although few studies have formally examined the clinical effects or metabolism of MDPV in humans, multiple first-hand reports and a handful of published case reports now exist [6-10]. Stimulant effects, an increase in libido, anxiety, hallucinations, and paranoid psychosis have been reported [6]. MDPV appears to undergo CYP450 2D6, 2C19, and COMT phase 1 metabolism into methylcatechol and pyrrolidine, which in turn are glucuronidated to allow for renal excretion [11, 12]. Although the mechanism of action is not fully elucidated at this time, a dopaminergic mechanism is hypothesized due to the chemical similarity to amphetamines and the behavioral effects of MDPV that mimic those of amphetamines which are known to affect dopaminergic pathways. Studies on other pyrovalerone analogs demonstrated potent inhibition of the dopamine and norepinephrine transporters responsible for reuptake of their corresponding neurotransmitters [13]. Further supporting this hypothesis are animal studies demonstrating MDPV increases dopamine levels in experimental mice and rats $[14,15]$.

Since late 2010, MDPV has been the most commonly detected $\beta$-keto phenylalkylamine in toxicologic analysis of "bath salts," "plant foods," or other legal highs in the USA. This differs from the European experience where mephedrone was most commonly detected in these substances $[1,9,16]$. This difference has an unknown clinical significance, as reports of $\beta$-keto phenylalkylamine use in the USA describe similar sympathomimetic effects of both mephedrone and MDPV, including agitation, hypertension, palpitations, hallucinations, and violent behavior; however, the US reports have differed in part from those from Europe by the increased prevalence of MDPV, as well as an increase in cases involving rhabdomyolysis.

We report a case of MDPV toxicity with symptoms consistent with Excited Delirium Syndrome (ExDS) followed by a sudden PEA arrest, return of spontaneous circulation, and subsequent development of coagulopathy, rhabdomyolysis, renal failure, hepatic failure, anoxic brain injury, and death. This is the first case in the medical literature to report death due to isolated MDPV intoxication. Toxicological analysis of serum and urine revealed MDPV as an isolated intoxicant. Additionally, while a death consistent with ExDS has been reported after mephedrone use, to our knowledge, this is the first reported MDPV-associated death [17].

ExDS is characterized by delirium with agitation, hyperthermia, tachypnea, tachycardia, a period of "giving up," or cessation of struggle, followed by cardiac arrest, as was seen in our patient [18]. ExDS most likely results from dysregulation of dopaminergic pathways. ExDS autopsy data have shown a decreased number of D3 dopamine receptors when compared to controls [19]. This suggests that ExDS patients may lack normal compensatory measures for dealing with rapid changes in dopamine levels. Furthermore, hypothalamic dopamine receptors are responsible for thermoregulation, and patients with ExDS have elevated heat shock proteins consistent with the clinically observed hyperthermia [19]. Hyperthermia in these patients is likely a sign of autonomic dysfunction that results in death in many ExDS patients. However, in patients that survive the initial arrest, such as the patient in this report, hyperthermia likely contributes to the coagulopathy, rhabdomyolysis, and multisystem organ failure that result in death after the initial insult. ExDS has been described with MDMA, cocaine, and amphetamine intoxication [20,21].

In our case, the patient's previous history of cocaine abuse may have also predisposed him to ExDS, as chronic cocaine abuse is known to alter neurotransmitters such as dopamine [22]. The dopaminergic effects of MDPV likely contributed to aberrant thermoregulation, and the sympathomimetic effects contributed to the agitation, delirium, and the cardiac arrest in this patient. Treatment for MDPV intoxication, along with other drugs in the stimulant class and patients with ExDS remains largely supportive, with the use of intravenous benzodiazepines, intravenous fluids, and cooling.

The patient in this case was also found to have trimethoprim present on serum and urine testing. This was quantified $(12 \mathrm{mcg} / \mathrm{mL}$ in urine and $2.2 \mathrm{mcg} / \mathrm{mL}$ in serum) and present in amounts that exceed previous reports of peak plasma levels in single one-time ingestions of $160 \mathrm{mg}$ doses [23]. Trimethoprim is not reported to cause stimulant effects with chronic use or in acute overdose. There are reports of hyperkalemia occurring in patients predisposed to potassium retention (underlying renal failure or concurrent diuretic use) [24, 25]. Our patient was not known to be taking a trimethoprim-containing medication, and personal communication with the laboratory performing the test (telephone correspondence with Dr. S. Kacinko, NMS Labs, August 2011) revealed an additional case where MDPV and trimethoprim were found together using similar testing methods. We hypothesize that this may be an adulterant in some formulations of "bath salts," including that which our patient consumed. It is therefore possible trimethoprim contributed to our patient's hyperkalemia, but we do not feel it is responsible for his ExDS symptoms or ultimate death.

We were initially perplexed as to why our patient's serum ethanol level continued to rise well after admission even with the initiation of hemodialysis; however, ethanol 
was not detected on a volatile alcohol screen performed on blood obtained during this time period. Most likely the serum ethanol levels quantified via the serum ethanol assay (Beckman UniCel DxC, Beckman Coulter Inc, Brea, CA) were falsely elevated due to interference from the patient's profoundly elevated lactate dehydrogenase (LDH) $(11,108$ IU/L, $19 \mathrm{~h}$ after presentation) and lactate levels $(10.7 \mathrm{mmol} /$ $\mathrm{L}, 19 \mathrm{~h}$ after presentation). This interference of LDH and lactate with serum ethanol determination has been described with multiple instruments/assays, including the methodology used to measure ethanol in this case [26-28]. These assays are based on the conversion of ethanol to acetylaldehyde by alcohol dehydrogenase reducing NAD to NADH in the process, which is quantitated by absorbance at $340 \mathrm{~nm}$ due to NADH formation. Assay interference occurs when excess LDH and lactate allow for conversion of NAD to NADH in a reaction unrelated to the presence of ethanol.

\section{Conclusion}

Recreational abuse of "bath salts" products continues despite legislative initiatives to prohibit its use. While this is the first reported case of death due to isolated recreational use of MDPV with confirmatory toxicological analyses, further characterization of symptoms related to intoxication and associated deaths is needed. This case highlights the need for emergency physicians and toxicologists to be vigilant in testing for novel drugs of abuse, as it allows for an improved understanding of the potential toxicity of these drugs and associated adulterants.

\section{References}

1. Spiller H, Ryan M, Weston R, Jansen J (2011) Clinical experience with and analytical confirmation of "bath salts" and "legal highs" (synthetic cathinones) in the United States. Clin Toxicol 49 (6):499-505

2. Yohannan J, Bozenko J (2010) The characterization of 3, 4-methylenedioxypyrovalerone (MDVP). Microgram Journal 7:21-15

3. 1-[(3, 4-Methylenedioxy)phenyl]-2-pyrrolidino-1 alkanones as stimulants (1969) Boehringer Ingelheim Study

4. Westphal F, Junge T, Rösner P, Sönnichsen F, Schuster F (2009) Mass and NMR spectroscopic characterization of 3,4methylenedioxypyrovalerone: a designer drug with alphapyrrolidinophenone structure. Forensic Sci Int 190(1-3):1-8

5. Uchiyama N, Kikura-Hanajiri R, Kawahara N, Goda Y (2008) Analysis of designer drugs detected in the products purchased in fiscal year 2006. Yakugaku Zasshi 128(10):1499-1505
6. Antonowicz J, Metzger A, Ramanujam S (2011) Paranoid psychosis induced by consumption of methylenedioxypyrovalerone: two cases. Gen Hosp Psychiatry 33(6):640.e5-6

7. Penders T, Gestring R (2011) Hallucinatory delirium following use of MDPV: "Bath Salts." Gen Hosp Psychiatry 30:525-526

8. Kriikku P, Wilhelm L, Schwarz O, Rintatalo J (2011) New designer drug of abuse: 3,4-methylenedioxypyrovalerone (MDPV) findings from apprehended drivers in Finland. Forensic Sci Int 210(1-3): 195-200

9. Centers for Disease Control and Prevention (CDC) (2011) Emergency department visits after use of a drug sold as "bath salts"-Michigan, November 13, 2010-March 31, 2011. MMWR Morb Mortal Wkly Rep 60(19):624-627

10. Smith C, Cardile C, Miller C (2011) Bath salts as a "legal high." Am J Med. doi:10.1016/j.amjmed.2011.03.014

11. Strano-Rossi S, Cadwallader AB, de la Torre X, Botre F (2010) Toxicological determination and in vitro metabolism of the designer drug methylenedioxypyrovalerone (MDPV) by gas chromatography/mass spectrometry and liquid chromatography/ quadrupole time-of-flight mass spectrometry. Rapid Commun Mass Spectrom 24(18):2706-2714

12. Meyer M, Du P, Schuster F, Maurer H (2010) Studies on the metabolism of the $\alpha$-pyrrolidinophenone designer drug methylenedioxy-pyrovalerone (MDPV) in rat and human urine and human liver microsomes using GC-MS and LC-highresolution MS and its detectability in urine by GC-MS. J Mass Spectrom 45(12):1426-1442

13. Meltzer P, Butler D, Deschamps J, Madras B (2006) 1-(4Methylphenyl)-2-pyrrolidin-1-yl-pentan-1-one (Pyrovalerone) analogs A promising class of monoamine uptake inhibitors. J Med Chem 49(4):1420-1432

14. Fuma T, Kodama T, Honda Y, Tanaka T, Kubo Y, Ohashi N et al (2009) Influence of methy lenedioxypyrovalerone on central nervous system using micro dialysis method. ChemBio Integrated Management 5:62-72

15. Nagai $F$ et al (2007) The effects of non-medically used psychoactive drugs on monoamine neurotransmission in rat brain. Eur J Pharmacol 559(2-3):132-137

16. McElrath K, O’Neill C (2011) Experiences with mephedrone preand post-legislative controls: perceptions of safety and sources of supply. Int J Drug Policy 22(2):120-127

17. Lusthof KJ, Oostinga R, Maesa A, Verschraagena M, Dijkhuizena A, Spronga AGA (2011) A case of extreme agitation and death after the use of mephedrone in the Netherlands. Forensic Sci Int 206(1-3):e93-e95

18. Takeuchi AT, Henderson S (2011) Excited delirium. West J Emerg Med 12(1):77-83

19. Mash D, Duque L, Pablo J, Qin Y, Adi N, Hearn W, Hyma B, Karch S, Druid H, Wetli C (2009) Brain biomarkers for identifying excited delirium as a cause of sudden death. Forensic Sci Int 190(1-3):e13-e19

20. Dar K, McBrien M (1996) MDMA induced hyperthermia: report of a fatality and review of current therapy. Intensive Care Med 22 (9):995-996

21. DiMaio TG, DiMaio VJ (2005) Excited delirium syndrome: cause of death and prevention. CRC Press, New York

22. Staley J, Welti C, Ruttenber A et al (1995) Altered dopaminergic synaptic markers in cocaine psychosis and sudden death. NIDA Res Monogr Series 153:491

23. Bach MC, Gold O, Finland M (1973) Absorption and urinary excretion of trimethoprim, sulfamethoxazole and trimethoprimsulfamethoxazole. J Infect Dis 128(Suppl):584-598 
24. Weir M, Juurlink D, Gomes T, Mamdani M, Hackam D, Jain A et al (2010) Beta-blockers, trimethoprim-sulfamethoxazole, and the risk of hyperkalemia in the elderly: a nested case-control study. Clin J Am Soc Nephrol 5:1544-1551

25. Lam N, Weir M, Juurlink D, Gunraj N, Gomes T, Mamdani M et al (2011) Hospital admissions for hyperkalemia with trimethoprimsulfamethoxazole: a cohort study using health care database codes for 393,039 older women with urinary tract infections. Am J Kidney Dis 57(3):521-523
26. Nine J, Moraca M, Virji M, Rao K (1995) Serum-ethanol determination: comparison of lactate and lactate dehydrogenase interference in three enzymatic assays. J Anal Toxicol 19:192-196

27. Gharapetian A, Holmes D, Urquhart N, Rosenerg F (2008) Dehydrogenase interference with enzymatic ethanol assays: forgotten but not gone. Clin Chem 54(7):1251-1252

28. King G, Bissell M (2011) Does your lab produce false positive ethanol results? AACC/CAP Serum Alcohol Proficiency Survey A12-C:2-4 